\title{
Book Review: Clinical training guide for the student music therapist (2nd edition)
}

\author{
Janelle Chambers ${ }^{1 *}$ \\ 1 Slippery Rock University, United States \\ *janellechambersloan@gmail.com
}

Received: 27 March 2018 ; Accepted: 6 June 2018 ; Published: 1 July 2018

Editor: Melody Schwantes

\section{Introduction}

A decade has gone by since the original publication (Wheeler, Shultis, \& Polen, 2005) of this text (Polen, Shultis, \& Wheeler, 2017). Much of this book has generally remained the same, making it a consistent and valuable resource for its readers, but the slight changes, additions, edits, and updated references have created a text that is more relevant for today's student music therapists and their supervisors.

I am reviewing this book with a mere 8 years of various professional clinical and supervision experience, as well as a nearly completed Masters in Music Therapy degree. While my combined years and hours of experience does not come close to the years and hours of experience of the well-established authors of this text, my experiences and professional perspectives are unique and hopefully contribute a valuable viewpoint. In recent years, my personal and professional worldview has embraced a stronger commitment to social justice and cultural responsiveness. Therefore, I have also included a section in this review that highlights and critiques elements of the book that may or may not address the social, cultural, and political implications and considerations that the student music therapist may encounter.

\section{Overview}

The first two chapters of the book provide a brief overview of theories, definitions, therapeutic processes, and considerations for the student music therapist. An important gem in this section is Bruscia's most recent definition of music therapy compared to his previous ones (pp. 5-6), demonstrating that worldviews can and should change over time. This sets an important example for the student music therapist to not become complacent or stagnant in their understanding or worldview of music therapy.

Students and supervisors will benefit greatly from the self-reflection assignments in these first two chapters, which encourage the student to start thinking about their own definitions, assumptions, experiences, priorities, values, and personal beliefs when it comes to music therapy. Developing a foundation of some of these principles will be critical as the student music therapist begins to understand and practice the other tools and resources found later in the book.

Chapter 3, "Essential Aspects of Becoming a Music Therapist: Academic, Clinical Training, and Related Areas," is a great addition to this book that was missing from the original text. It is equipped with many resources and provides directions on ways to access those resources. It encourages the student music therapist's awareness of and responsibility to their professional organization and standards of practice. 
The section on supervision in this chapter is valuable, but could have placed more emphasis on the importance of continuing supervision outside of the educational setting. Supervision is a part of the packaged deal in a student's clinical training but often does not get pursued once the student finishes their curriculum and enters the professional field. I realize that professional entry level techniques are not the focus of this book, but stressing supervision as a professional may help some students more greatly recognize its importance. Perhaps this suggestion is more of a prompt for professional organizations to provide an accessible resource on finding music therapy supervisors, especially for entry level professionals.

In Chapter 4, "The Process of Planning for Music Therapy," there was a focus on the student spending time reflecting on their own understanding of therapy, relationship to music, and reactions and assumptions about clients. It is clear that these authors find this to be an important beginning step in the student's personal and clinical development.

Chapters 5 \& 6, "Assessments" and "Goals and Objectives," are both written very thoroughly and clearly and seem to be applicable to various philosophies and methodologies. The types of assessments described on pages 37-39 are helpful for students in understanding and framing the different purposes and uses of music therapy assessments. The examples of assessments provided are useful and well-organized. There are several additional examples and references mentioned in the paragraphs, should the student/supervisor feel that the provided example assessments are not quite appropriate for the particular setting and/or clientele.

Both of these chapters place an emphasis on music's role in the assessment and goal writing processes. As student music therapists move into the professional field, they may find themselves having to squeeze music therapy into the molds of other disciplines, causing them to strip away the uniqueness of what they do to fit or accommodate other professions. The more students learn and internalize the essence of using music in music therapy assessments and goal-writing, the more they will hold onto it as they are faced with those barriers and limitations later in their career.

Chapter 7 combined two chapters from the first edition, "Planning Music Therapy Strategies" and "Organizing the Session" into "Planning and Implementing Music Therapy Strategies". A useful resource from this chapter is Bruscia's Strategy/Activity Form (Table 7.1, p. 72) that could be used to keep a record of activities and experiences to increase and improve the student's clinical repertoire. Another valuable section is the emphasis on the environment in the therapeutic space (pp. 78-83). This section encourages the student to think through each physical and musical element that they are bringing into the session and provides pros, cons, and considerations of each.

Chapters 8-11 explore the improvising, re-creative, compositional, and receptive music experiences most commonly used in music therapy. Each of these chapters provides a thorough overview with multiple references and literature regarding each type of music experience and their application to different populations, as well various considerations and tips for each. In terms of populations mentioned, there was nothing specific on adolescent psych or hospice. It is possible that the authors do not think that these are populations that most undergraduate clinical students are assigned. There is a very important section in chapter 11 regarding copyright laws and boundaries to consider when bringing in personal music into the therapy setting that may not always get emphasized in clinical training. The assignments at the end of each chapter continue to encourage reflexivity, while also building on the material in previous chapters and promote "real world" application.

The chapter titled "Further Considerations" (Chapter 12) is the part of the book that puts together the student's developing personal theory with the developing skills from previous chapters. A treasured element of this chapter is the inclusion of Kenny's suggested steps on developing theory on page 137.

The tables of different levels of music therapy practice (pp. 133-135) are very useful in describing the different types of music therapy within different settings and with different people. This is helpful in being sure that the most appropriate care is being 
provided for the context and that the therapist is not crossing boundaries or practicing outside of their scope of practice. I do worry that by numbering the levels $1,2,3$ that a hierarchy is placed on them, suggesting that some types of music therapy are better or more superior than others, when really they are just different and more or less suitable depending on the situation and/or setting.

The breakdown and combination of facilitating both verbal and musical responses in Chapter 13 is informative and pertinent. Bruscia's techniques are very nicely summarized and organized in this section. There is also a return to the essence and emphasis on music, which once again brings music back into the heart of what we do. Similarly, Chapter 14, "The Role of Music," referenced many music-centered theories and reflects on the ways music represents and is used in relationship to societal structures.

The next two chapters (Chapter $15 \& 16$ ) explore useful references and considerations for working in group and individual music therapy, such as length of stay, formation of groups, structure, stages of development, boundaries, and the inclusion of the client(s) in therapeutic decisions. The following chapter on documentation (Chapter 17) is also thorough and provides several examples, definitions, and assignments that will be helpful for the student music therapist in a variety of settings and stages of their training.

The final chapter, "Self-Assessment of the Music Therapist," is a very important chapter and hopefully reminds the reader that self-assessment should be an ongoing process, not just an undergraduate educational one. The sections of this chapter mostly focus on assessing the clinical skills of the student, and once again put music at the core with the section titled "Using Music for Self-Assessment."

This chapter could have also included a section on self-care. Research indicates that music therapists who experience burnout were not provided education on recognizing the triggers and symptoms of burnout in their clinical training, and that they often felt burnt out before ever entering the field due to the intensity of their clinical training (Chang, 2014). Again, perhaps this is not the text for this topic, but including it as an important part of clinical training and providing self-care resources and references could have been added to this section.

\section{Sociocultural Elements}

Throughout this entire book, reflexivity is encouraged. The assignments, as well as other areas of the text, place a great deal of emphasis on the development of personal views and values, as well as sitting with contradicting and confusing thoughts and feelings. The authors never claim that the techniques and considerations are the "be all, end all" in music therapy. They never give "right or wrong" ways of "doing" music therapy, nor do they attempt to persuade the student into practicing one way or another. They constantly challenge the student music therapist to thoroughly think through clinical decisions and actions before carrying them out, and remind the reader that this is simply an introductory book and that they should continue to read, learn, explore, challenge, question, and critically reflect on their experiences.

This type of reflexive, transformative thinking and self-reflection is a critical first step in becoming a more culturally responsive music therapist (Hadley \& Norris, 2016). This book has multiple nods to this type of thinking and exploration, while also could have benefitted from additional resources and assignments regarding sociocultural issues. For example, in the "Ethical Considerations" sections of Chapter 4 (pp. 30-31) and Chapter 12 (pp. 138-139), the student music therapist is asked to explore their own personal ethical standards based on their own cultural values. An extra assignment reflecting on personal cultural values could have been included in these chapters, as this is critical in how we understand, treat, assess, and evaluate ourselves, others, and the therapeutic process.

Cultural considerations were present in the "Assessment" chapter (p. 42) and the "Goals and Objectives" chapter (pp. 68-69). Highlighted in these sections is the collab- 
oration with the client to establish assessments and goals, which is a beautiful example of ways to create more meaningful and culturally sensitive music therapy experiences. What could be added to these sections is a component about how the therapist's internal biases, assumptions, and worldviews could cause potential issues when facilitating assessments or writing goals and objectives.

As musical instruments were discussed in Chapter 7 (pp. 79-82), there should have been some encouragement for the student music therapist to be educated and curious about the cultural background and implications of instruments and music that they use in their sessions. Perhaps this is not the book for this type of exploration; however, it may be less instinctual, or not happen at all, if this consideration is not practiced as a student. It may have been helpful for this chapter to provide some resources that promote the student's cultural awareness in an effort to reduce potential cultural appropriation in the music therapy setting.

In the "Connections through Music" section of Chapter 14 (pp. 150-151), the authors reflect on the cultural roles and implications of music within society, moving the power of music therapy to outside of the clinical treatment walls. Perhaps more emphasis could have been placed on how those roles and implications are very different from culture to culture, identity to identity, and that the therapist needs to practice and be committed to being ever more conscious of this and not to assume or project their own relationship with music and culture onto that of their clients.

This type of cultural self-reflection and self-assessment could possibly have been included in Chapter 18, as it may be helpful for the student music therapist to start practicing assessing their personal psychosocial elements, such as assumptions, values, and biases regarding race, gender, sexuality, disability, age, etc. As mentioned previously, if this is not practiced or explored at this level, it may never be.

\section{Conclusion}

As I was reading through this book, there were multiple times that I started to make a note about an area, section, or idea that I felt was missed or overlooked, only to find it on the next page and/or chapter. This simply proves the comprehensiveness and thoroughness of this entire text. This book should be read and re-read throughout the various stages of a student's clinical training. One's personal and clinical experiences change the way these chapters are read and understood. When a person is "doing" music therapy, they are doing all of the things mentioned in this book, all at the same time. It is impossible to isolate only one element, read about that element, practice that element, become proficient in it, and then move onto the next. Therefore, it is also not feasible to be proficient in all areas from the start, and yet the student music therapist needs to start somewhere. This text is an excellent place to start, and an excellent resource to return to and reference throughout their clinical training and development.

I am reviewing this text as a professional clinician, a graduate student, and a supervisor, and I am still getting something out of it. It is good to refresh, reconnect, and revisit concepts that were instilled in us as students with a different worldview and understanding of ourselves, our clients, and of music therapy.

Overall, this is an excellent resource. Most everything that is needed for the student music therapist is all in one place in this text, with guidelines and ways to explore additional materials and topics as needed. I see it as being an invaluable resource for supervisors, educators, practicing clinicians, and students alike.

\section{References}

Chang, K. (2014). An opportunity for positive change and growth: Music therapists' experiences of burnout. Canadian Journal of Music Therapy, 20, 64-85.

Hadley, S., \& Norris, M. (2016). Musical multicultural competency in music therapy: The first step. Music Therapy Perspectives, 34, 129-137, https://dx.doi.org/10.1093/mtp/miv045. 
Polen, D., Shultis, C., \& Wheeler, B. (2017). Clinical training guide for the student music therapist (2nd ed.). Dallas: Barcelona Publishers.

Wheeler, B., Shultis, C., \& Polen, D. (2005). Clinical training guide for the student music therapist. Gilsum, NH: Barcelona Publishers. 